Nota

\title{
QUALIDADE DE MANGAS CULTIVADAS NO ESTADO DE SÃO PAULO ( $\left.{ }^{\mathbf{1}}\right)$
}

\author{
JULIANA ALTAFIN GALLI $\left({ }^{2 *}\right)$; MARCOS DONISETI MICHELOTTO $\left({ }^{2}\right)$; \\ LUIS CLÁUDIO PATERNO SILVEIRA $\left({ }^{3}\right)$; ANTÔNIO LÚCIO MELO MARTINS $\left({ }^{2}\right)$
}

\section{RESUMO}

A manga (Mangifera indica L.) é uma das frutas mais consumidas no mundo. A escolha da variedade a ser plantada deve estar relacionada com as preferências do mercado consumidor, que incluem frutos com elevada porcentagem de polpa e alto teor de sólidos solúveis, propriedades de grande importância para o processamento industrial e para o mercado interno e externo. Foram avaliadas a qualidade dos frutos de 39 variedades de manga (Adams, Alda, Brasil, Cacipura, Carabao, Carrie, Castro, Edward, Eldon, Família, Foice, Haden, Haden 2H, Haden TR, IAC 10 Bourbon, Itamarati, Joe Welch, Julima, Lima Peru, M20-222 Winter, Mabrooca, Neves, Palmer, Pele de Moça, Petacon, Pope, Regina, Rocha, Rosa, Rubi, Sensation, Smith, Surpresa, Torbet, Torrinha, Ubá, Wesley, White Langra e Zill) da coleção do Pólo Apta Centro-Norte, localizado em Pindorama, São Paulo, objetivando a obtenção de informações sobre a qualidade dos frutos produzidos nesta região. Vinte frutos de cada variedade foram colhidos maduros e avaliados quanto à massa fresca, massa da casca, massa do caroço, porcentagens de polpa, casca e caroço, teor de sólidos solúveis totais (SST) e infestação por mosca-das-frutas. Para a análise dos dados de porcentagem de polpa e SST utilizou-se delineamento em blocos casualizados, e as médias foram comparadas pelo teste de Tukey a $5 \%$ de probabilidade. Com base nos resultados, pôde-se concluir que a variedade Smith destacou-se pelos frutos com maior massa total (694 g), maior porcentagem de polpa $(85,7 \%)$, menor porcentagem de caroço $(6,3 \%)$ e maior teor de SST $\left(24,9^{\circ}\right.$ Brix $)$.

Palavras-chave: Mangifera indica L., rendimento, sólidos solúveis, mosca-das-frutas.

\section{ABSTRACT \\ MANGOES QUALITY CULTIVATED IN SÃO PAULO STATE}

The mango (Mangifera indica L.) is one of the most consumed fruits in the world. The choice of variety for cultivation should be related with preferences of the consuming market, that include fruits with larger pulp percentage and larger tenor of total soluble solids, properties of great importance for industrial processing and for internal and external markets. The overall quality of fruits from 39 mango varieties (Adams, Alda, Brasil, Cacipura, Carabao, Carrie, Castro, Edward, Eldon, Família, Foice, Haden, Haden 2H, Haden TR, IAC 10 Bourbon, Itamarati, Joe Welch, Julima, Lima Peru, M20-222 Winter, Mabrooca, Neves, Palmer, Pele de Moça, Petacon, Pope, Regina, Rocha, Rosa, Rubi, Sensation, Smith, Surpresa, Torbet, Torrinha, Ubá, Wesley, White Langra and Zill) from the collection of Pólo Apta Centro-Norte, located at Pindorama, São Paulo State, was appraised, with the objective of gathering information regarding fruits produced in this area. Twenty fruits of each variety were picked ripe traits such as fresh weight, weight of the peel, weight of the pit, pulp, peel and pit percentages, tenor of total soluble solids (SST) and infestation for fruit-flyes were evaluated. A design in randomized blocks was used for the analysis of the data of pulp percentage and SST. Averages were compared by the Tukey test of 5\% of probability. Based on results, it can be concluded that the variety Smith stands out for exhibiting fruits with larger total weight $(694 \mathrm{~g})$, larger pulp percentage $(85,7 \%)$, smaller pit percentage $(6,3 \%)$ and larger tenor of SST $\left(24,9^{\mathrm{O}}\right.$ Brix $)$, adequate for industrial processing and for internal and external markets.

Key words: Mangifera indica L., yield, soluble solids, fruit flies.

$\left({ }^{1}\right)$ Recebido para publicação em 11 de junho de 2007 e aceito em 29 de janeiro de 2008.

$\left({ }^{2}\right)$ Pólo Apta Centro-Norte (DDD/APTA/SAA), Caixa Postal 24, 15830-000 Pindorama (SP). E-mail: julianagalli@apta.sp.gov.br $\left(^{*}\right)$ Autora correspondente; michelotto@apta.sp.gov.br; lmartins@apta.sp.gov.br

$\left({ }^{3}\right)$ Universidade Federal de Lavras, UFLA, Caixa Postal 3037, 37200-000 lavras (MG). E-mail: lcpsilveira@ufla.br 


\section{Introdução}

A manga (Mangifera indica L.) é uma das frutas mais comercializadas no mundo. A procura pelo produto tem aumentado bastante nos mercados interno e externo, alcançando preços compensatórios.

O Brasil está entre os nove principais países produtores de manga do mundo, com uma área plantada de 67 mil hectares. A comercialização da manga no mercado interno brasileiro centraliza-se em uma única variedade, a norte-americana Tommy Atkins, que representa 79\% da área plantada no País (PINTO, 2002).

Diversos fatores estão relacionados com a escolha da variedade de manga a ser plantada, destacando-se as preferências do mercado consumidor, o potencial produtivo da variedade para uma determinada região, as limitações fitossanitárias e de pós-colheita, e principalmente do potencial de mercado da variedade em médio prazo. Sendo a mangueira uma planta com longo período juvenil, a escolha da variedade errada poderá ocasionar enormes prejuízos em curto prazo. Assim, a escolha da variedade é considerada um dos fatores econômicos mais importantes para o estabelecimento competitivo da mangicultura (Costa e SANTOS, 2004).

Entre as características que podem servir de suporte para a avaliação da qualidade de manga, estão a aparência externa, o sabor, o odor, o teor de fibras, a textura, o valor nutritivo, o tamanho, a massa e a forma. Essas características poderão variar muito, conforme a variedade e o local de cultivo, além de ocorrer alterações sensíveis durante o processo de amadurecimento (ARAÚJo, 2004).

Em tese, o consumidor não se preocupa se a variedade de manga é mais produtiva ou mais resistente à determinada doença, mas sim com a qualidade do fruto que vai consumir. $\mathrm{O}$ sabor, o rendimento e a textura da polpa são qualidades muito importantes para a escolha do consumidor (PINTO, 2002). Para o produtor, porém, é importante que, além das características organolépticas, a variedade tenha boas característica de cultivo.

Devido à importância da mangueira no contexto nacional e à grande procura por informações técnicas por parte dos produtores regionais, este trabalho teve como objetivo avaliar as características dos frutos de diferentes variedades de mangueira para seleção e recomendação, visando à comercialização nos mercados interno e externo.

\section{Material e Métodos}

Foram avaliadas 39 variedades de manga: Adams, Alda, Brasil, Cacipura, Carabao, Carrie,
Castro, Edward, Eldon, Família, Foice, Haden, Haden $2 \mathrm{H}$, Haden TR, IAC 10 Bourbon, Itamarati, Joe Welch, Julima, Lima Peru, M20-222 Winter, Mabrooca, Neves, Palmer, Pele de Moça, Petacon, Pope, Regina, Rocha, Rosa, Rubi, Sensation, Smith, Surpresa, Torbet, Torrinha, Ubá, Wesley, White Langra e Zill, de área experimental localizada a $21^{\circ} 13^{\prime}$ de latitude sul; $48^{\circ}$ $55^{\prime}$ de longitude oeste; com temperatura média anual de $22,8^{\circ} \mathrm{C}$, precipitação média anual de $1390,3 \mathrm{~mm}$ e umidade relativa média anual de $71,6 \%$.

As variedades analisadas foram enxertadas sobre a variedade Manila (Carabao). As mudas foram plantadas em 1998, em espaçamento $10 \times 10 \mathrm{~m}$, três árvores de cada variedade. Os tratos culturais realizados seguiram as técnicas normalmente recomendadas para a cultura na região, exceto as podas de manejo da planta.

Na safra 2005-2006, foram colhidos 20 frutos maduros das três plantas de cada variedade. A maioria das variedades classificaram-se em "da estação" (maturação em dezembro/janeiro) e as variedades Alda, Cacipura, Castro, Palmer, Regina e Sensation foram classificadas como tardias (maturação em fevereiro/março). Em cada fruto foram avaliados os seguintes parâmetros: massa total (g), massa do caroço (g), massa da casca (g) e massa da polpa (g), utilizando balança comercial com precisão de $5 \mathrm{~g}$. A partir desses dados, foram calculadas as porcentagens de casca, caroço e polpa em relação à massa total. As variedades foram classificadas em relação à massa média dos frutos, aplicando-se a classificação estabelecida por DonADIo et al. (1982): frutos pequenos $(<250 \mathrm{~g})$; médios $(250$ a $350 \mathrm{~g})$; grandes (350 a $500 \mathrm{~g}$ ) e muito grandes ( $>500 \mathrm{~g}$ ).

O teor de Sólidos Solúveis Totais (SST) foi determinado em refratômetro manual, com precisão de duas casas decimais, fazendo-se leituras em duas ou três gotas de suco da polpa, retiradas de dois cortes longitudinais do fruto, um de cada lado da semente. Os resultados foram expressos em ${ }^{\circ} \mathrm{Brix}$.

A suscetibilidade ao ataque de mosca-dasfrutas foi avaliada em condições de infestação natural de campo, cortando-se longitudinalmente cada um dos 20 frutos em ambos os lados do caroço para exame visual. Frutos com qualquer grau de infestação foram considerados infestados pela mosca, e calculou-se a porcentagem de frutos infestados (CARVALHo et al., 2004).

Os dados de porcentagem de polpa e SST foram avaliados seguindo delineamento experimental em blocos casualizados. Foi realizada análise de variância, sendo as médias comparadas pelo teste de Tukey a $5 \%$ de probabilidade de erro. 


\section{Resultados e Discussão}

De acordo com a massa dos frutos, obteve-se a seguinte classificação: os frutos das variedades Adams, Alda, Carabao, Neves, Pele de Moça, Petacon, Ubá e Wesley foram classificados como pequenos; os frutos das variedades Cacipura, M20-222 Winter, Rocha, Rubi, Sensation e Torrinha foram classificados como médios; os frutos das variedades Brasil, Carrie, Castro, Edward, Eldon, Haden 2H, Haden TR, IAC 100 Bourbon, Itamarati, Julima, Mabrooca, Pope, Rosa, Surpresa, Torbet, White Langra e Zill foram classificados como grandes, e os frutos das variedades Família, Foice, Haden, Joe Welch, Lima Peru, Palmer, Regina e Smith foram classificados como muito grandes (Tabela 1). Segundo Botrel (1994), a preferência do consumidor brasileiro não é regulada pelo tamanho do fruto, mas sim pelo seu sabor.

No entanto, para o mercado externo, exige-se que os frutos tenham determinados pesos. No caso dos Estados Unidos os frutos devem ter entre 250 e $450 \mathrm{~g}$. Para a Europa, exige-se que os frutos tenham até 650 g (Genú e Pinto, 2002).

As variedades Smith, Edward, Haden TR, Joe Welch, Família, Haden 2H, Torbet, Mabrooca, Haden, Eldon, Lima Peru, White Langra, Pope, Julima, Zill e Surpresa, classificadas como grandes e muito grandes, atingiram rendimentos de polpa superior a $80 \%$ (Tabela 2). Segundo Ascenso et al. (1981), a porcentagem de polpa nos frutos é uma característica de grande importância, uma vez que constitui a parte comestível, admitindo-se como uma classe alta em polpa aquela que perfaz $85 \%$ da massa do fruto até a muito pobre, com $70 \%$.

O tamanho do caroço perfazendo o máximo de $10 \%$ da massa do fruto é um dos requisitos básicos para que uma variedade tenha importância comercial (Young e SAuls, 1979; DonAdio, 1980). Nas variedades, cujos rendimentos de polpa foram superiores a $80 \%$, as porcentagens de caroço foram de $6,3 \%$ a $9,3 \%$. No entanto, os resultados do experimento revelaram que as variedades Carrie, Rubi, Rocha, IAC 100 Bourbon, Cacipura, Rosa, Palmer, Regina, Petacon, Castro, Brasil, Foice, Adams, Neves, Sensation, Ubá, Carabao, Alda e Pele de Moça têm porcentagens de caroço de 10,1\% até $20,5 \%$ em relação à massa do fruto (Tabela 2), característica indesejável a uma variedade.

Durante o processo de amadurecimento da manga, o nível de amido decresce e há rápido acúmulo se sólidos solúveis. Segundo Natividad Ferrer (1987) citado por Botrel (1994), a porcentagem de sólidos solúveis constatada na manga varia de 6,65 a 21,9 ${ }^{\circ}$ Brix, dependendo da variedade e do estádio de maturação do fruto.

As variedades Smith, Ubá, Carabao, IAC 11 Bourbon, Carrie, Castro, Cacipura, M20-222 Winter, Wesley, Edward e Neves proporcionaram os maiores valores de ${ }^{\circ}$ Brix (acima de $17^{\circ}$ ), considerado ideal para boa receptividade do fruto. Pode se considerar que a amplitude de variação de SST do experimento foi grande, uma vez que variou de $24,9^{\circ}$ Brix, na variedade Smith, a $12,5^{\circ}$ Brix, na variedade Brasil (Tabela 3).

BOtrel (1994) relata que tanto a acidez dos frutos quanto o teor de sólidos solúveis totais podem influenciar na aceitação das cultivares. BLEINROTH et al. (1985), ao avaliarem 22 cultivares de mangas do município de Tietê (SP), observaram teor médio de sólidos solúveis de $15,85^{\circ} \mathrm{Brix}$, dentro de um intervalo de 12,1 a $19{ }^{\circ}$ Brix. Carvalho et al. (2004) verificaram índices de sólidos solúveis de 16,6 a 20,2 Brix em mangas da região de Votuporanga (SP).

Verificou-se nas variedades comportamento diferente quanto a infestação dos frutos pela moscadas-frutas (Tabela 3). De modo geral, o nível de infestação pôde ser considerado baixo, com os maiores valores atingindo $20 \%$, nas variedades da estação Torbet e Rocha. Nas variedades tardias Castro, Regina e Palmer havia, respectivamente, 0, 5\% e 15\% de frutos infestados, contrariando os resultados de SETOGUCHI (1991), ao verificar que as variedades tardias são mais suscetíveis ao ataque da praga.

O grande número de espécies de mosca-dasfrutas existente, a variação ambiental e as épocas de incidência dificultam os estudos sobre resistência varietal de fruteiras a esse tipo de inseto. Estudos realizados no Brasil atestam que a infestação e a freqüência com que são constatados frutos atacados nos diferentes locais pesquisados podem ser usadas como critério para se considerar o fruto como hospedeiro principal ou secundário. Em coletas de manga e de citros (Citrus spp.) efetuadas nesses estudos, observou-se que, em menos da metade dos locais, as amostras estavam infestadas. Por esse motivo, tais frutos foram considerados como hospedeiros secundários. Por outro lado, em frutos silvestres, como pitanga (Eugenia uniflora L.), uvaia (E. pyriformis Cambess) e guabiroba (Campomanesia xantocarpa O. Berg), o índice de infestação foi elevado, circunstância que caracteriza tais frutos como hospedeiros principais (BRANCO et al., 1999). 
Tabela 1. Médias da massa total, massa da casca, massa do caroço e rendimento de polpa, de 20 frutos maduros de 39 variedades de manga cultivadas em Pindorama (SP), em 2005/2006

\begin{tabular}{|c|c|c|c|c|}
\hline Variedades & Massa total & Massa casca & Massa caroço & Massa polpa \\
\hline & & 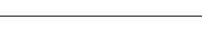 & 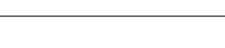 & \\
\hline Adams & 246,75 & 25,25 & 31,00 & 190,50 \\
\hline Alda & 246,25 & 31,25 & 39,00 & 176,00 \\
\hline Brasil & 397,00 & 35,25 & 42,50 & 319,25 \\
\hline Cacipura & 322,50 & 35,25 & 34,00 & 253,25 \\
\hline Carabao & 202,50 & 26,00 & 29,75 & 146,75 \\
\hline Carrie & 350,50 & 36,00 & 34,75 & 279,75 \\
\hline Castro & 353,00 & 38,50 & 39,00 & 275,50 \\
\hline Edward & 452,50 & 35,00 & 29,50 & 388,00 \\
\hline Eldon & 424,25 & 40,50 & 39,25 & 344,50 \\
\hline Família & 545,75 & 52,25 & 42,50 & 451,00 \\
\hline Foice & 641,00 & 68,75 & 77,50 & 494,75 \\
\hline Haden & 600,25 & 57,75 & 50,00 & 492,50 \\
\hline Haden $2 \mathrm{H}$ & 461,25 & 40,75 & 40,00 & 380,50 \\
\hline Haden TR & 480,75 & 38,00 & 35,75 & 407,00 \\
\hline IAC100Bourbon & 370,25 & 39,75 & 38,00 & 292,50 \\
\hline Itamarati & 364,00 & 38,50 & 35,75 & 289,75 \\
\hline Joe Welch & 587,00 & 53,50 & 45,25 & 488,25 \\
\hline Julima & 486,75 & 50,50 & 41,00 & 395,25 \\
\hline Lima Peru & 527,50 & 55,25 & 44,50 & 427,75 \\
\hline M20-222 Winter & 293,75 & 36,50 & 26,25 & 231,00 \\
\hline Mabrooca & 421,75 & 37,00 & 38,75 & 346,00 \\
\hline Neves & 243,50 & 32,00 & 30,25 & 181,25 \\
\hline Palmer & 536,25 & 50,75 & 56,50 & 429,00 \\
\hline Pele de moça & 147,00 & 26,50 & 29,50 & 91,00 \\
\hline Petacon & 245,25 & 35,25 & 27,25 & 182,75 \\
\hline Pope & 448,00 & 51,25 & 29,75 & 367,00 \\
\hline Regina & 604,25 & 70,25 & 64,50 & 469,50 \\
\hline Rocha & 292,50 & 42,00 & 29,75 & 220,75 \\
\hline Rosa & 366,50 & 39,50 & 39,75 & 287,25 \\
\hline Rubi & 282,50 & 27,75 & 28,00 & 226,75 \\
\hline Sensation & 291,25 & 27,50 & 36,25 & 227,50 \\
\hline Smith & 694,00 & 55,25 & 43,25 & 595,50 \\
\hline Surpresa & 491,25 & 49,50 & 43,25 & 398,50 \\
\hline Torbet & 489,00 & 50,50 & 35,00 & 403,50 \\
\hline Torrinha & 330,25 & 41,75 & 28,00 & 260,50 \\
\hline Ubá & 138,75 & 17,25 & 19,00 & 102,50 \\
\hline Wesley & 207,00 & 21,25 & 32,50 & 153,25 \\
\hline White Langra & 463,25 & 47,25 & 39,75 & 376,25 \\
\hline Zill & 481,25 & 48,50 & 42,75 & 390,00 \\
\hline Média & 398,13 & 41,17 & 38,18 & 318,78 \\
\hline CV (\%) & 11,75 & 13,62 & 10,20 & 12,97 \\
\hline
\end{tabular}


Tabela 2. Proporções dos componentes polpa, caroço e casca nos frutos de 39 variedades de manga cultivadas em Pindorama (SP), 2005/06

\begin{tabular}{|c|c|c|c|c|}
\hline Variedades & Polpa & Caroço & Casca & Caroço + Casca \\
\hline & 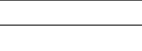 & 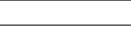 & 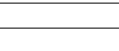 & 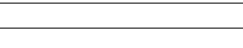 \\
\hline Smith & 85,7 a & 6,3 & 8,0 & 14,3 \\
\hline Edward & $85,4 \mathrm{ab}$ & 6,6 & 7,9 & 14,6 \\
\hline Haden TR & $84,2 \mathrm{a}-\mathrm{c}$ & 7,7 & 8,2 & 15,8 \\
\hline Joe Welch & 83,0 a-d & 7,8 & 9,3 & 17,7 \\
\hline Família & 82,6 a-d & 7,9 & 9,5 & 17,4 \\
\hline Haden $2 \mathrm{H}$ & 82,3 a-e & 8,8 & 8,9 & 17,7 \\
\hline Torbet & 82,3 a-f & 7,2 & 10,5 & 17,7 \\
\hline Mabrooca & 81,8 a-f & 9,3 & 8,9 & 18,2 \\
\hline Haden & $81,7 \mathrm{a}-\mathrm{g}$ & 8,5 & 9,8 & 18,3 \\
\hline Eldon & 81,2 a-g & 9,3 & 9,5 & 18,8 \\
\hline Lima Peru & $80,9 \mathrm{~b}-\mathrm{g}$ & 8,6 & 10,5 & 19,1 \\
\hline White Langra & $80,8 \mathrm{~b}-\mathrm{g}$ & 8,7 & 10,4 & 19,2 \\
\hline Pope & $80,8 \mathrm{~b}-\mathrm{g}$ & 7,5 & 11,7 & 19,2 \\
\hline Julima & $80,7 \mathrm{~b}-\mathrm{g}$ & 8,6 & 10,7 & 19,3 \\
\hline Zill & $80,6 \mathrm{c}-\mathrm{g}$ & 9,1 & 10,3 & 19,4 \\
\hline Surpresa & $80,6 \mathrm{c}-\mathrm{g}$ & 9,0 & 10,5 & 19,4 \\
\hline Rubi & $79,6 \mathrm{c}-\mathrm{h}$ & 10,2 & 10,2 & 20,4 \\
\hline Carrie & $79,3 \mathrm{~d}-\mathrm{h}$ & 10,1 & 10,6 & 20,7 \\
\hline Brasil & $79,3 \mathrm{~d}-\mathrm{h}$ & 11,4 & 9,3 & 20,7 \\
\hline Itamarati & $79,2 \mathrm{~d}-\mathrm{h}$ & 9,9 & 10,9 & 20,8 \\
\hline Palmer & $79,2 \mathrm{~d}-\mathrm{h}$ & 10,8 & 9,9 & 20,8 \\
\hline Torrinha & $78,7 \mathrm{~d}-\mathrm{i}$ & 8,5 & 12,7 & 21,3 \\
\hline IAC100Bourbon & $78,7 \mathrm{~d}-\mathrm{i}$ & 10,4 & 10,9 & 21,3 \\
\hline M20-222 Winter & $78,3 \mathrm{~d}-\mathrm{j}$ & 9,1 & 12,6 & 21,7 \\
\hline Cacipura & $78,2 \mathrm{~d}-\mathrm{j}$ & 10,7 & 11,1 & 21,8 \\
\hline Rosa & $78,2 \mathrm{~d}-\mathrm{j}$ & 10,8 & 10,9 & 21,7 \\
\hline Sensation & 77,6 e-j & 12,7 & 9,7 & 22,4 \\
\hline Castro & $77,5 \mathrm{f}-\mathrm{j}$ & 11,3 & 11,2 & 22,5 \\
\hline Adams & $77,0 \mathrm{~g}-\mathrm{j}$ & 12,6 & 10,4 & 23,0 \\
\hline Regina & 77,0 g-j & 10,9 & 12,1 & 23,0 \\
\hline Foice & $77,0 \mathrm{~g}-\mathrm{j}$ & 12,2 & 10,9 & 23,0 \\
\hline Rocha & $75,2 \mathrm{~h}-\mathrm{k}$ & 10,3 & 14,5 & 24,8 \\
\hline Petacon & 74,4 i-k & 11,1 & 14,5 & 25,6 \\
\hline Neves & 74,1 i-k & 12,6 & 13,3 & 25,9 \\
\hline Wesley & $73,8 \mathrm{jk}$ & 15,9 & 10,3 & 26,2 \\
\hline Ubá & $73,6 \mathrm{jk}$ & 13,9 & 12,5 & 26,4 \\
\hline Carabao & $71,5 \mathrm{k}$ & 15,1 & 13,4 & 28,5 \\
\hline Alda & $71,0 \mathrm{k}$ & 16,1 & 12,9 & 29,0 \\
\hline Pele de moça & 60,91 & 20,5 & 18,6 & 39,1 \\
\hline Média & 78,6 & 10,46 & 10,97 & 21,43 \\
\hline $\mathrm{CV}(\%)$ & 2,17 & 7,60 & 11,59 & 7,94 \\
\hline
\end{tabular}

Médias seguidas pela mesma letra não diferem entre si, pelo teste de Tukey, a 5\% de probabilidade. 
Tabela 3. Média de Grau Brix e quantidade de frutos infestados pela mosca-das-frutas em 20 frutos maduros de 39 variedades de manga cultivadas em Pindorama (SP), em 2005/2006

\begin{tabular}{|c|c|c|c|}
\hline \multirow[t]{2}{*}{ Variedades } & \multirow[t]{2}{*}{ Graus Brix } & \multicolumn{2}{|c|}{ Frutos infestados } \\
\hline & & $\mathrm{n} .^{\circ}$ & $\%$ \\
\hline Smith & $24,9 \mathrm{a}$ & 3 & 15 \\
\hline Ubá & $22,1 \mathrm{ab}$ & 0 & 0 \\
\hline Carabao & $20,2 \mathrm{a}-\mathrm{c}$ & 1 & 5 \\
\hline IAC 100 Bourbon & $19,1 \mathrm{a}-\mathrm{c}$ & 2 & 10 \\
\hline Carrie & 18,9 a-c & 0 & 0 \\
\hline Castro & 18,5 a-c & 0 & 0 \\
\hline Cacipura & $18,2 \mathrm{a}-\mathrm{c}$ & 0 & 0 \\
\hline M20-222 Winter & 17,7 a-c & 2 & 10 \\
\hline Wesley & $17,1 \mathrm{a}-\mathrm{c}$ & 0 & 0 \\
\hline Edward & $17,1 \mathrm{a}-\mathrm{c}$ & 0 & 0 \\
\hline Neves & $17,1 \mathrm{a}-\mathrm{c}$ & 0 & 0 \\
\hline Zill & $16,1 \mathrm{bc}$ & 0 & 0 \\
\hline Surpresa & $16,1 \mathrm{bc}$ & 1 & 5 \\
\hline Pope & $16,0 \mathrm{bc}$ & 1 & 5 \\
\hline Pele de Moça & $15,8 \mathrm{bc}$ & 2 & 10 \\
\hline Adams & $15,7 \mathrm{bc}$ & 0 & 0 \\
\hline Eldon & $15,7 \mathrm{bc}$ & 0 & 0 \\
\hline Lima Peru & $15,4 \mathrm{bc}$ & 0 & 0 \\
\hline Rubi & $15,4 \mathrm{bc}$ & 0 & 0 \\
\hline Família & $15,3 \mathrm{bc}$ & 0 & 0 \\
\hline Torrinha & $15,2 \mathrm{bc}$ & 2 & 10 \\
\hline Alda & $15,2 \mathrm{bc}$ & 2 & 10 \\
\hline Petacon & $15,1 \mathrm{bc}$ & 3 & 15 \\
\hline Joe Welch & $15,0 \mathrm{bc}$ & 0 & 0 \\
\hline Palmer & $14,8 \mathrm{bc}$ & 3 & 15 \\
\hline Mabrooca & $14,7 \mathrm{bc}$ & 1 & 5 \\
\hline Itamarati & $14,8 \mathrm{bc}$ & 2 & 10 \\
\hline Sensation & $14,4 \mathrm{bc}$ & 0 & 0 \\
\hline White Langra & $14,3 \mathrm{bc}$ & 1 & 5 \\
\hline Regina & $14,0 \mathrm{bc}$ & 1 & 5 \\
\hline Haden $2 \mathrm{H}$ & $14,0 \mathrm{bc}$ & 3 & 15 \\
\hline Haden & $13,8 \quad b c$ & 2 & 10 \\
\hline Haden TR & $13,7 \mathrm{bc}$ & 0 & 0 \\
\hline Foice & $13,7 \mathrm{bc}$ & 2 & 10 \\
\hline Torbet & $13,2 \mathrm{c}$ & 4 & 20 \\
\hline Julima & $13,0 \mathrm{c}$ & 1 & 10 \\
\hline Rocha & $13,0 \mathrm{c}$ & 4 & 20 \\
\hline Rosa & $12,7 \mathrm{c}$ & 2 & 10 \\
\hline Brasil & $12,5 \mathrm{c}$ & 2 & 10 \\
\hline Média & 15,9 & - & - \\
\hline CV (\%) & 19,54 & - & - \\
\hline
\end{tabular}

Médias seguidas pela mesma letra não diferem entre si, pelo teste de Tukey, a $5 \%$ de probabilidade.

\section{Conclusão}

Entre as variedades estudadas, a "Smith" destaca-se pelos frutos com maior massa total (694 g), maior porcentagem de polpa $(85,7 \%)$, menor porcentagem de caroço $(6,3 \%)$ e maior teor de sólidos solúveis totais $\left(24,9^{\circ}\right.$ Brix $)$, propriedades de grande importância para o processamento industrial e para o mercado consumidor da fruta in natura interno e externo.

\section{Referências}

ARAÚJO, J.L.P. Mercado e comercialização da manga. In: MOUCO, M.A.C. Cultivo da Mangueira. Petrolina: Embrapa Semi-Árido, Sistemas de Produção, 2. Versão eletrônica, 2004.

ASCENSO, J.C.; MILHEIRO, A.V.; MOTA, M.I.; CABRAL, M. Seleção preliminar da mangueira. Pesquisa Agropecuária Brasileira, Brasília, v.16, n.3, p.417-429, 1981.

BLEINROTH, E.W.; FIGUEIREDO, I.B.; VEIGA, A.A.; SOARES, N.B.; MEDINA, J.C.; SABINO, J.C. Avaliação de novas cultivares de manga para industrialização. I. Análise das características físico e químicas da matéria-prima. Boletim do ITAL, Campinas, v.22, n.2, p.217-246, 1985.

BOTREL, N. Manga: Variedades, Qualidade e Tecnologia PósColheita. Informe Agropecuário, Belo Horizonte, v.17, n. 179, p.55-60, 1994.

BRANCO, E.S.; VENDRAMIN, J.D.; DENARDI, F. Resistência às moscas-das-frutas em fruteiras. In: MALAVASI, A.; ZUCCHI, R.A. (Ed.). Moscas-das-frutas de importância econômica no Brasil: conhecimento básico e aplicado. Ribeirão Preto: Holos, 1999. p.161-167.

CARVALHO, C.R.L.; ROSSETTO, C.J.; MANTOVANI, D.M.B.; MORGANO, M. A.; CASTRO, J.V.; BORTOLETTO, N. Avaliação de cultivares de mangueira selecionadas pelo Instituto Agronômico de Campinas comparadas a outras de importância comercial. Revista Brasileiras de Fruticultura, Jaboticabal, v.26, n.2, p.264-271, 2004.

COSTA, J.G.; SANTOS, C.A.F.Cultivares. In: MOUCO, M.A.C. Cultivo da Mangueira. Petrolina: Embrapa Semi-Árido, Sistemas de Produção, 2. Versão eletrônica, 2004.

DONADIO, L.C. Cultura da mangueira. Piracicaba: Livroceres, 1980.72p.

DONADIO, L.C.; SOARES, N.B.; MORAES, L. G.; XAVIER, N.J.D.; SCALOPI, E.J.; PIZA JUNIOR, C.T. Características de algumas variedades de mangueira cultivadas no Estado de São Paulo. São Paulo: CATI, 1982. 16p. (CATI. Boletim Técnico, 171).

GENÚ, P.J.C.; PINTO, A.C.Q. (Ed.). A cultura da mangueira. Brasília: EMBRAPA, 2002, 452p. 
PINTO, A.C.Q. A produção, o consumo e a qualidade da manga no Brasil. Revista Brasileira de Fruticultura, Cruz das Almas, v.24, n.3, p. 597, 2002

YOUNG, T.W.; SAULS, J.W. The mango industry in Florida. Gainesville: University of Florida, 1979, 70p. (Bulletin, 189)

SETOGUCHI, A.K. Resistência varietal da manga aos danos de mosca-das-frutas Anastrepha obliqua (Mcquart, 1835) (Diptera: Tephritidae). 1991. 51f. Monografia (Graduação em Agronomia) - Faculdade de Ciências Agrárias e Veterinárias, Universidade Estadual Paulista, Jaboticabal, 1991. 